\title{
ТЕМПЕРАТУРНЫЕ ЗАВИСИМОСТИ КОЭФФИЦИЕНТОВ ТЕПЛОПРОВОДНОСТИ ПОЛИМЕРНЫХ МИКРО- И НАНОКОМПОЗИТОВ ДЛЯ ТЕПЛООБМЕННЫХ АППАРАТОВ
}

Долинский А.А. ${ }^{1}$, академик НАН Украины, Фиалко Н.М. ${ }^{1}$, член-корреспондент НАН Украины, Динжос P.В. ${ }^{2}$, канд. физ.-мат. наук, Навродская Р.А. ${ }^{1}$, канд. техн. наук

${ }^{1}$ Институт технической теплофизики НАН Украины, ул. Желябова, 2 а, Киев, 03680, Украина

${ }^{2}$ Николаевский национальный университет им. В.А.Сухомлинского, ул. Никольская, 24, Николаев, 540030, Украина

Наведено результати експериментальних досліджень залежності від температури коефіцієнтів теплопровідності полімерних мікро- i нанокомпозитів, призначених для виготовлення теплообмінного обладнання. Представлено дані щодо врахування цієї залежності при визначенні складів композитів 3 заданими теплопровідними властивостями. Обговорюються особливості механізмів теплопереносу в полімерах, наповнених вуглецевими нанотрубками.

Библ. 12 , рис. 4 , табл. 2.
Приведены результаты экспериментальных исследований зависимости от температуры коэффициентов теплопроводности полимерных микро- и нанокомпозитов, предназначенных для изготовления теплообменного оборудования. Представлены данные, касающиеся учета этой зависимости при определении составов композитов с заданными теплопроводящими свойствами. Обсуждаются особенности механизмов теплопереноса в полимерах, наполненных углеродными нанотрубками.
The results of experimental investigations of the temperature dependence of the heat-conductivity coefficients of polymer micro- and nanocomposites for the manufacture of heat exchange equipment are given. The data relating to the account of this dependence in determining the composition of composites with desired heat-conducting properties are presented. The features of the heat transfer mechanisms in polymers filled with carbon nanotubes are discussed.

Ключевые слова: полимерные микро- и нанокомпозиты, коэффициент теплопроводности, углеродные нанотрубки, механизмы теплопроводности, теплообменники.

$T$ - температура, K;

$T_{1}, T_{2}$ - температура на горячем и холодном конце УHT, K;

$t$ - температура, ${ }^{\circ} \mathrm{C}$;

$t_{\min }, t_{\max }-$ минимальная и максимальная темпера-

тура исследуемых температурных диапазонов, ${ }^{\circ} \mathrm{C}$;

$\lambda$ - коэффициент теплопроводности, Вт/(м·К); $\Delta \lambda=\lambda_{\min }-\lambda_{\max }-$ отличие значений $\lambda$, отвечающих температурам $t_{\min }$ и $t_{\max }, \mathrm{BT} /(\mathrm{M} \cdot \mathrm{K})$;

$\delta \lambda=\frac{\Delta \lambda}{\lambda_{\max }} \cdot 100 \%-$ относительное отличие значений

\section{Введение}

Все более широкое использование полимерных микро- и нанокомпозитов для создания теп лообменных поверхностей обусловливает необходимость получения уточненных данных об их $\lambda, \%$

$\omega-$ массовая доля наполнителя, $\%$;

$\Delta \omega=\omega_{\max }-\omega_{\min }-$ отличие значений $\omega$, отвечающих температурам $t_{\max }$ и $t_{\min }, \%$;

$\delta \omega=\frac{\Delta \omega}{\omega_{\min }} \cdot 100 \%-$ относительное отличие значений $\omega, \%$;

ПК - поликарбонат;

ПММА - полиметилметакрылат;

ПП - полипропилен;

ПЭ - полиэтилен;

УНТ - углеродные нанотрубки.

теплофизических характеристиках [1-7]. Особый интерес представляет знание температурных зависимостей таких характеристик в рабочих диапазонах температур соответствующих теплообменных аппаратов. При этом для рассматриваемой 
ситуации в комплексе теплофизических свойств полимерных композиционных материалов, в первую очередь, выделяется их коэффициент теплопроводности, значения которого в большой мере определяют эффективность теплообменных устройств. Зависимости от температуры коэффициентов теплопроводности композитов являются, в частности, базой для обоснованного выбора их составов, отвечающих заданным теплопроводящим свойствам.

Настоящая статья посвящена, главным образом, экспериментальным исследованиям температурных зависимостей коэффициентов теплопроводности ряда полимерных микро- и нанокомпозитов, предназначенных для изготовления теплообменного оборудования различного назначения. При этом определенное внимание уделяется установлению особенностей влияния учета данных зависимостей при нахождении составов композитов с заданными значениями коэффициентов теплопроводности. Обсуждаются также вопросы, связанные с механизмами теплопроводности в рассматриваемых композитах.

\section{Постановка задачи и методика проведения исследований}

Данная работа касается экспериментального определения зависимости от температуры коэффициента теплопроводности композитов на основе ПЭ, ПММА, ПП и ПК, наполненных УНТ или микрочастицами алюминия. В ходе исследований максимальное содержание наполнителей достигало $10 \%$. Получение композитов основывалось на использовании метода смешения компонентов в расплаве с применением дискового екструдера [8]. При определении коэффициентов теплопроводности композитов использовалась методика, детальное описание которой приводится в [9].

В рамках настоящей работы ставилась также задача сопоставления полученных зависимостей $\lambda=f(T)$ для композитов на основе различных полимерных матриц, наполненных УНТ или микрочастицами алюминия. Кроме того в задачу исследования входило проведение сравнительного анализа данных о составах композитов с фиксированными величинами $\lambda$ в условиях, когда определение этих составов осуществлялось с учетом и без учета температурной зависимости коэффициентов теплопроводности.
Обсуждению подлежали также вопросы, касающиеся механизмов фононного теплопереноса в полимерах, наполненных УНТ.

\section{Результаты исследований и их анализ}

На рис. 1, 2, 3 и в табл. 1 представлены характерные результаты выполненных экспериментальных исследований, касающиеся определения температурных зависимостей коэффициентов теплопроводности рассматриваемых композитов. Рис. 1, 2 иллюстрируют данные об изменении $\lambda$ с температурой для полимеров, наполненных микрочастицами алюминия и УНТ соответственно, при различных значениях массовой доли наполнителя $\omega$. На рис. 3 приведены зависимости $\lambda=f(\omega)$ для полимеров, наполненных микрочастицами алюминия, при фиксированных значениях температуры. В табл. 1 для всех исследуемых композитов представлены значения наибольших абсолютных $\Delta \lambda$ и относительных $\delta \lambda$ отличий $\lambda$ в рассматриваемых диапазонах температур.

Как следует из полученных данных, для всех рассматриваемых композитов, за исключением двух, значения коэффициентов теплопроводности с ростом температуры уменьшаются. Для композитов на основе ПК, наполненных микрочастицами алюминия, и на основе ПП, наполненных УНТ, наблюдается обратная картина. Причем, в последнем случае изменение $\lambda$ во всем рассматриваемом диапазоне температур оказывается пренебрежимо малым.

Что же касается величин отличий $\Delta \lambda$ в значениях коэффициента теплопроводности, отвечающих наименьшей и наибольшей из температур рассматриваемого интервала, то как показали выполненные исследования, они зависят от типа матрицы и наполнителя, а также от массовой доли последнего.

Остановимся вначале на рассмотрении соответствующих данных для полимеров, наполненных микрочастицами алюминия. Как видно из табл. 1 , в этом случае значения $\Delta \lambda$ могут достигать $2,3 \mathrm{BT} /(\mathrm{M} \cdot \mathrm{K})$, что отвечает величине $\delta \lambda$, равной $10,1 \%$. То есть относительное отклонение величин коэффициентов теплопроводности, отвечающих минимальной и максимальной из рассматриваемых температур, здесь могут быть весьма заметными.

Согласно полученным данным для полиме- 
ров, наполненных микрочастицами алюминия, значения $\Delta \lambda$ существенно зависят от типа полимерной матрицы. По мере убывания $\Delta \lambda$ композитов рассматриваемые полимерные матрицы ранжируются следующим образом: ПММА, ПЭ,
ПП и ПК. При использовании данных матриц максимальные в исследуемом диапазоне $\omega$ величины $\Delta \lambda$ составляют 2,$3 ; 2,0 ; 1,1$ и $-0,5 \mathrm{BT} /(\mathrm{M} \cdot \mathrm{K})$, что отвечает значениям $\delta \lambda$, равным 10,$1 ; 8,9 ; 4,2$ и $-2,7 \%$.
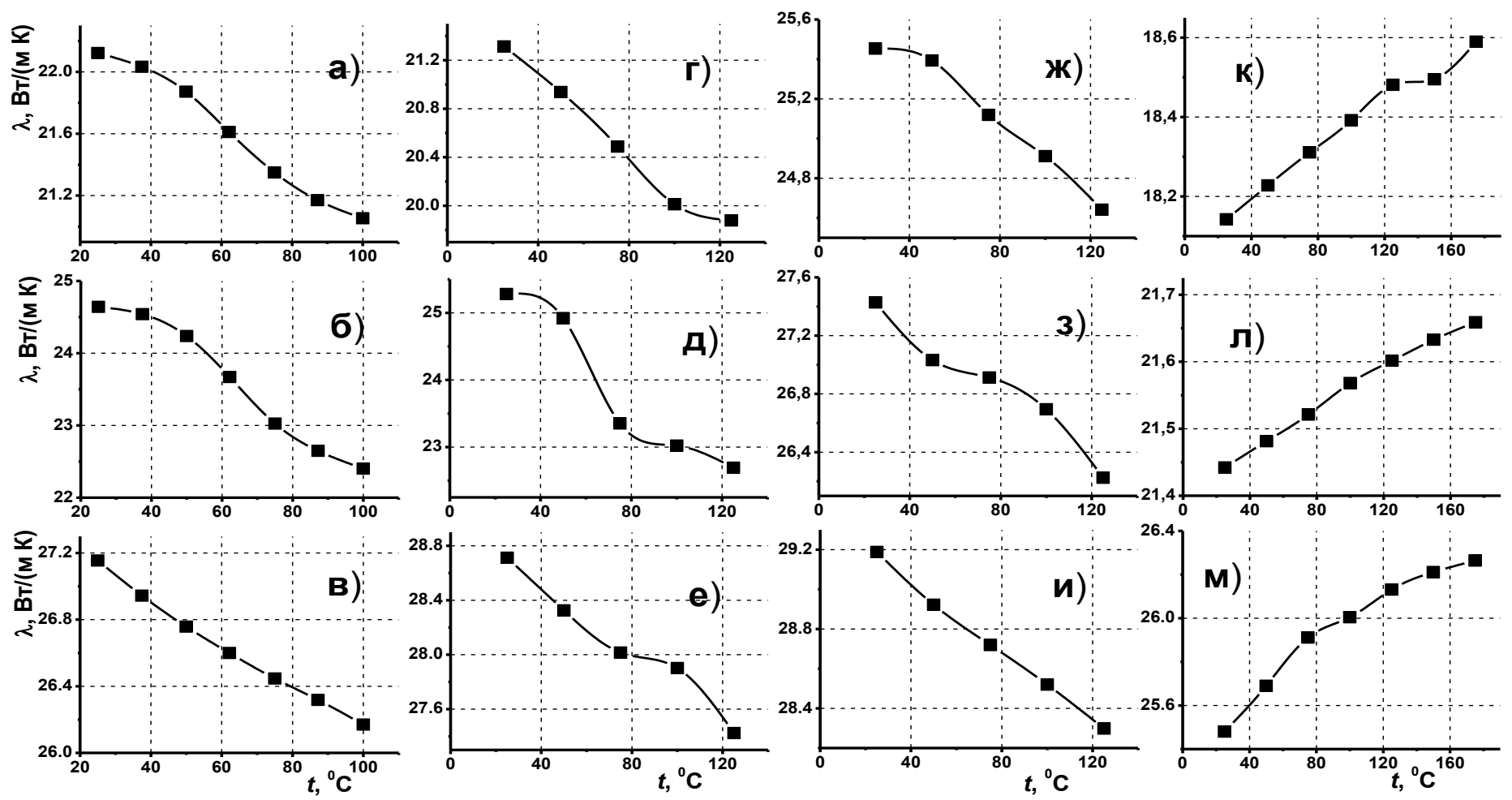

Рис. 1. Зависимость коэффициентов теплопроводности от температуры для композитов, наполненных микрочастицами алюминия, при использовании в качестве полимерных матриц ПЭ (а, б, в), ПММА (2, д, e), ПП (юс, з, и) и ПК (к, л, м) для различных значений $5 \%(a, 2, ж c, \kappa) ; 7 \%(\sigma, \delta, 3, \pi)$ и $10 \%(6, e, u, м)$.

Обращает на себя внимание также тот факт, что при наполнении полимеров микрочастицами алюминия величины $\delta \lambda$ существенно меняются с изменением массовой доли наполнителя. При этом зависимость $\delta \lambda=f(\omega)$ носит экстремальный характер. Как следует из приведенных данных, для композитов на основе ПЭ, ПММА и ПП с повышением $\omega$ от 5 до $7 \%$ величина $\delta \lambda$ растет, достигая максимального значения, и затем падает при дальнейшем увеличении $\omega$. Для композитов на основе ПК поведение $\delta \lambda$ с изменением $\omega$ имеет противоположный характер. Здесь с ростом $\omega$ от 5 до $8 \%$ значение $\delta \lambda$ уменьшается и далее с увеличением $\omega$ повышается.

Перейдем далее к рассмотрению величин от- клонений $\delta \lambda$ для полимеров, наполненных УНТ. Согласно полученным данным для этой ситуации значения $\delta \lambda$ сравнительно невелики и не превышают 4,1 \%. При этом наибольшие величины $\delta \lambda$ отвечают использованию в качестве полимерной матрицы ПК $(\delta \lambda \leq 4,1 \%)$, несколько меньшие ПММА $(\delta \lambda \leq 3,9 \%)$ и ПЭ $(\delta \lambda \leq 3,5 \%)$ и наименьшие - ПП $(|\delta \lambda| \leq 0,3 \%)$.

Что касается зависимости $\delta \lambda$ от содержания УНТ в композите, то здесь в целом наблюдается тенденция к уменьшению $\delta \lambda$ с ростом $\omega$. Так, для композитов на основе ПЭ при $\omega=3 \%, 5 \%$ и $10 \%$ соответствующие отклонения $\delta \lambda$ равны $3,5 \%, 1,9 \%$ и $1,1 \%$. 

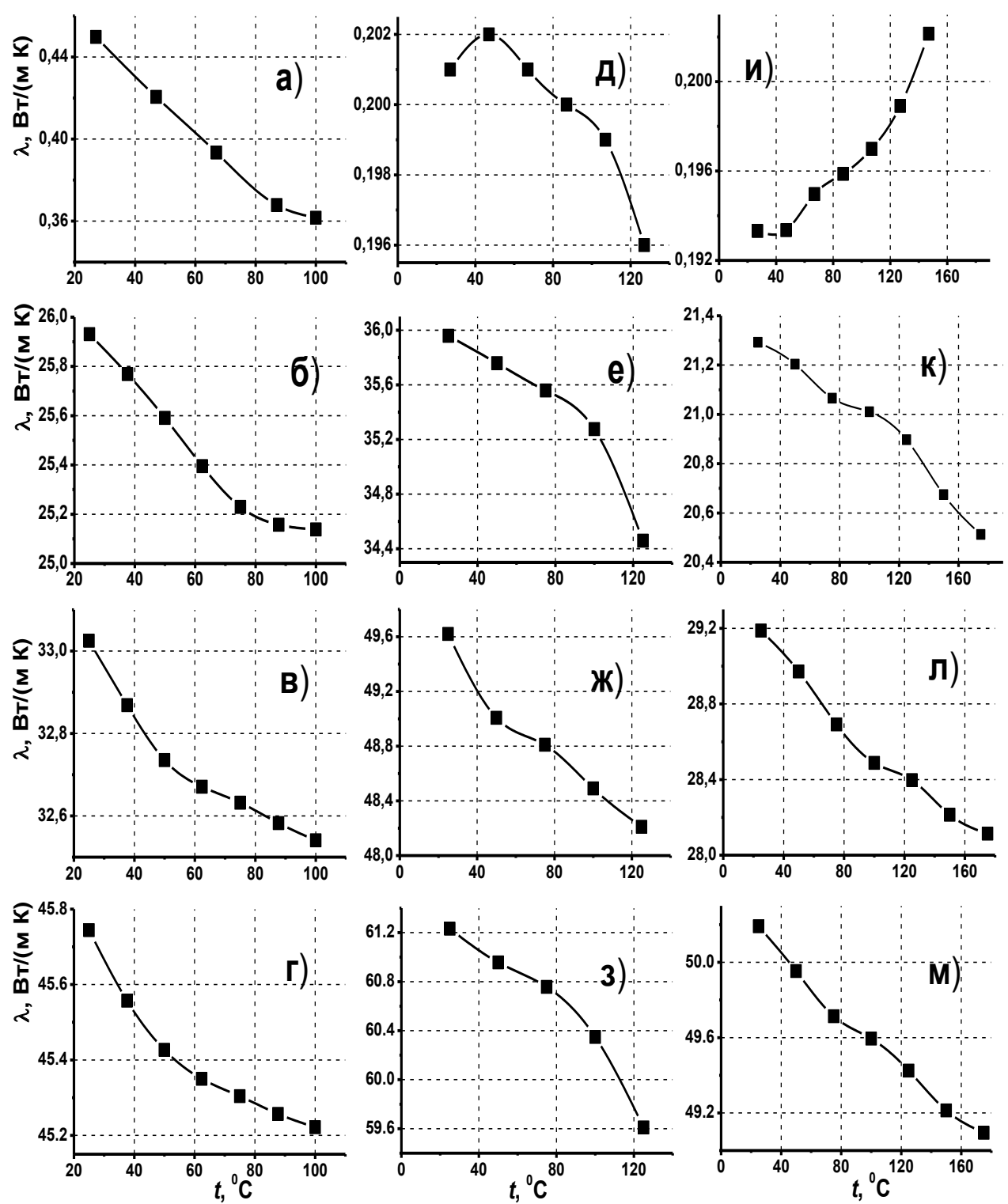

Рис. 2. Зависимость коэффициентов теплопроводности от температуры для ПЭ (а), ПММА (ठ), ПК (и) и композитов, наполненных УНТ, на основе ПЭ $(б, 6,2)$, ПММА (е, ж, з) и ПК (к, л, м) при различных значениях $\omega:$ $3 \%(\sigma, e, \kappa) ; 5 \%(6, ж, \pi) ; 10 \%(2,3, \mu)$.

Таким образом, результаты выполненных исследований свидетельствуют о том, что значения $\lambda$, полученные при температуре $t_{\min }\left(t_{\min }=\right.$ $=25{ }^{0} \mathrm{C}$ ), заметно отличаются от таковых при $t=t_{\max }$ только для композитов ПММА и ПЭ, наполненных алюминием. При этом наибольшие отклонения указанных величин $\lambda$ наблюдаются при массовой доле наполнителя, равной примерHо $7 \%$.

На основе полученных зависимостей $\lambda=f(\omega)$ при различных значениях температуры могут быть определены составы композитов с заданными теплопроводящими свойствами для данных температур. Как очевидно, при разработке таких составов должны использоваться значения $\lambda$ композитов, найденные по той из температур рассматриваемого диапазона, при которой коэффициенты теплопроводности являются наименьшими. Однако, как правило, выбор составов композитов осуществляется по наименьшей $t_{\min }$ из темпера- 
тур исследуемого интервала, равной температуре окружающей среды. Такой подход может приводить к тому, что требуемые значения $\lambda$ не будут обеспечиваться во всем диапазоне рабочих температур рассматриваемых композитов. В этой
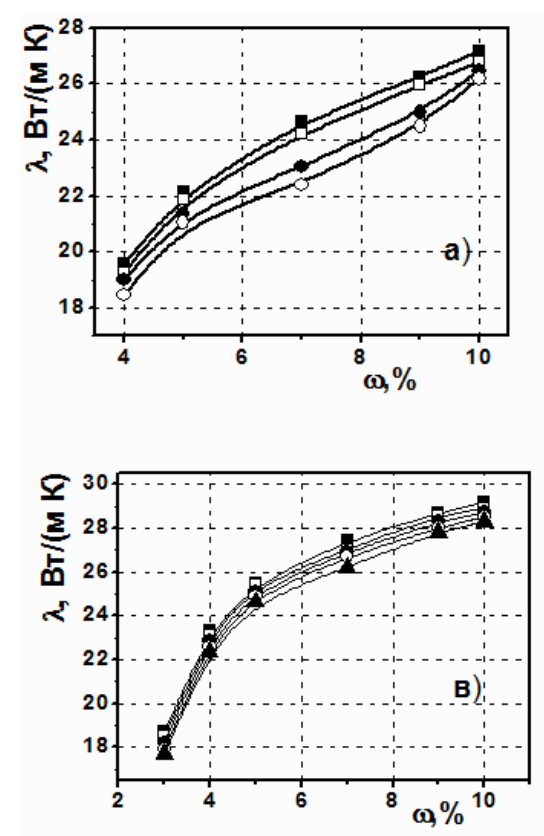

связи представляет интерес выполнить оценку влияния температурной зависимости коэффициентов теплопроводности композитов на выбор их требуемого состава, отвечающего заданным теплопроводящим свойствам.
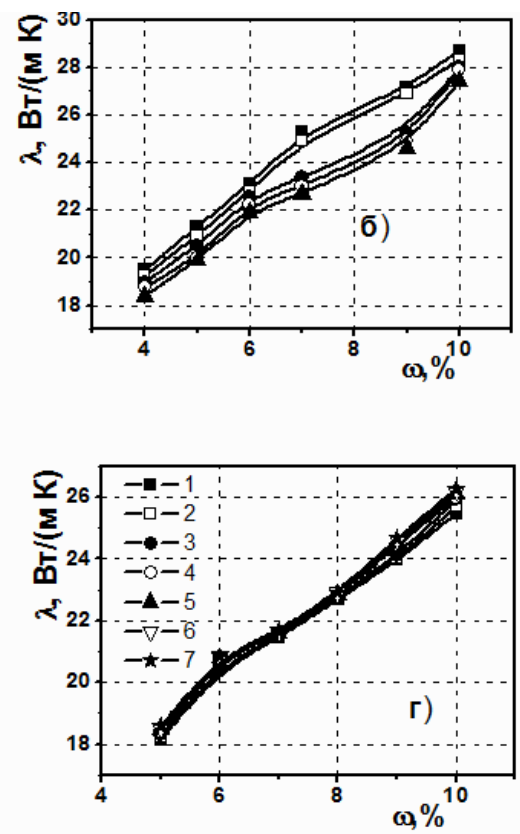

Рис. 3. Зависимость коэффициентов теплопроводности композитов от массовой доли микрочастиц алюминия при использовании в качестве полимерных матриц ПЭ (а), ПММА (б), ПП (в) и ПК (2) для различных значений температуры: $1-25^{\circ} \mathrm{C} ; 2-50{ }^{\circ} \mathrm{C} ; 3-75^{\circ} \mathrm{C} ; 4-100{ }^{\circ} \mathrm{C} ; 5-125^{\circ} \mathrm{C} ; 6-150^{\circ} \mathrm{C} ; 7-175^{\circ} \mathrm{C}$.

Табл. 1. Абсолютные $\Delta \lambda$ и относительные $\delta \lambda$ отличия значений $\lambda$, отвечающих минимальной и максимальной из температур исследуемого интервала, для композитов, наполненных УНТ или микрочастицами алюминия, при использовании в качестве полимерных матриц ПЭ, ПММА, ПП и ПК для различных величин $\omega$

\begin{tabular}{|c|c|c|c|c|c|c|c|}
\hline \multicolumn{4}{|c|}{ Наполнитель алюминий } & \multicolumn{4}{|c|}{ Наполнитель УНТ } \\
\hline $\begin{array}{c}\text { Тип поли- } \\
\text { мерной матрицы }\end{array}$ & $\omega, \%$ & $\Delta \lambda, \mathrm{BT} /(\mathrm{M} \cdot \mathrm{K})$ & $\delta \lambda, \%$ & $\begin{array}{c}\text { Тип поли- } \\
\text { мерной матрицы }\end{array}$ & $\omega, \%$ & $\Delta \lambda, \mathrm{BT} /(\mathrm{M} \cdot \mathrm{K})$ & $\delta \lambda, \%$ \\
\hline \multirow{3}{*}{ ПЭ } & 5 & 1,2 & 5,8 & \multirow{3}{*}{ ПЭ } & 3 & 0,8 & 3,5 \\
\hline & 7 & 2,0 & 8,9 & & 5 & 0,6 & 1,9 \\
\hline & 10 & 1,0 & 3,8 & & 10 & 0,5 & 1,1 \\
\hline \multirow{3}{*}{ ПММА } & 5 & 1,4 & 7,0 & \multirow{3}{*}{ ПММА } & 3 & 1,3 & 3,9 \\
\hline & 7 & 2,3 & 10,1 & & 5 & 1,3 & 2,7 \\
\hline & 10 & 1,3 & 4,7 & & 10 & 1,8 & 3,0 \\
\hline \multirow{3}{*}{ ПП } & 5 & 0,9 & 3,7 & \multirow{3}{*}{ ПП } & 3 & $-0,1$ & $-0,3$ \\
\hline & 7 & 1,1 & 4,2 & & 5 & $-0,1$ & $-0,2$ \\
\hline & 10 & 0,9 & 3,2 & & 10 & $-0,1$ & $-0,2$ \\
\hline \multirow{3}{*}{ ПК } & 5 & $-0,5$ & $-2,7$ & \multirow{3}{*}{ ПК } & 3 & 0,8 & 4,1 \\
\hline & 7 & $-0,2$ & $-0,9$ & & 5 & 1,0 & 3,5 \\
\hline & 10 & $-0,8$ & $-3,0$ & & 10 & 1,1 & 2,2 \\
\hline
\end{tabular}


Для всех разрабатываемых композиционных материалов, за исключением двух, наименьшие значения $\lambda$ имеют место при максимальной из рассматриваемых температур, и соответственно по ней должны определяться необходимые составы композитов. Для композита на основе ПК, наполненного частицами алюминия, и для композита на основе ПП, наполненного УНТ, наименьшие значения $\lambda$, напротив, наблюдаются при минимальной из исследуемого диапазона температур, то есть температуре окружающей среды $t=25^{\circ} \mathrm{C}$. В этом случае заданные значения $\lambda$ следует определять по данной температуре.

В табл. 2 представлены данные о величинах отличий составов рассматриваемых компози- тов, найденных по наибольшей и наименьшей из температур рассматриваемого интервала. Как видно из табл. 2 , отличия $\Delta \omega$ в целом существенно выше при наполнении полимеров микрочастицами алюминия, чем при их наполнении УНТ. Так, при использовании в качестве наполнителя микрочастиц алюминия эти отличия достигают для композитов на основе ПЭ $30,8 \%$, на основе ПММА - 29,7 \% и на основе ПК - 19,6 \%. Иными словами, для обеспечения требуемых значений коэффициентов теплопроводности во всем рабочем диапазоне температур композитов массовые доли алюминия, найденные по температуре окружающей среды, должны быть увеличены на указанную величину отличий $\Delta \omega$.

Табл. 2. Величины массовой доли наполнителей (УНТ и микрочастицы алюминия), найденные по минимальной и максимальной температуре исследуемого диапазона, и отличия этих долей для композитов на основе ПЭ, ПММА, ПП и ПК

\begin{tabular}{|c|c|c|c|c|c|c|c|c|c|c|c|}
\hline \multicolumn{6}{|c|}{ Наполнитель Al } & \multicolumn{6}{|c|}{ Наполнитель УНТ } \\
\hline $\begin{array}{l}\lambda \\
\mathrm{BT} /(\mathrm{M} \cdot \mathrm{K})\end{array}$ & $\begin{array}{l}\text { Тип } \\
\text { матрицы }\end{array}$ & $\omega_{\min }, \%$ & $\omega_{\max }, \%$ & $\Delta \omega, \%$ & $\delta \omega, \%$ & $\begin{array}{l}\lambda, \\
\mathrm{BT} /(\mathrm{M} \cdot \mathrm{K})\end{array}$ & $\begin{array}{l}\text { Тип } \\
\text { матрицы }\end{array}$ & $\omega_{\min }, \%$ & $\omega_{\max }, \%$ & $\Delta \omega, \%$ & $\delta \omega, \%$ \\
\hline \multirow{4}{*}{20} & ПЭ & 4,2 & 4,7 & 0,5 & 11,9 & \multirow{3}{*}{20} & ПЭ & 2,75 & 2,8 & 0,05 & 1,8 \\
\hline & ПММА & 4,3 & 5,0 & 0,7 & 16,3 & & ПММА & 2,4 & 2,45 & 0,05 & 2,1 \\
\hline & ПП & 3,3 & 3,5 & 0,2 & 6,1 & & ПК & 3,0 & 3,1 & 0,1 & 3,3 \\
\hline & ПК & 5,9 & 5,7 & $-0,2$ & $-3,4$ & \multirow{3}{*}{30} & ПЭ & 4,1 & 4,3 & 0,2 & 4,9 \\
\hline \multirow{4}{*}{22} & ПЭ & 5,1 & 6,4 & 1,3 & 25,5 & & ПММА & 2,7 & 2,8 & 0,1 & 3,7 \\
\hline & ПММА & 5,4 & 6,3 & 0,9 & 16,7 & & ПК & 5,1 & 5,4 & 0,3 & 5,9 \\
\hline & ПП & 3,7 & 4,0 & 0,3 & 8,1 & \multirow{3}{*}{40} & ПЭ & 7,5 & 7,7 & 0,2 & 2,7 \\
\hline & ПК & 7,4 & 7,2 & $-0,2$ & $-2,7$ & & ПММА & 3,5 & 3,7 & 0,2 & 5,7 \\
\hline \multirow{4}{*}{24} & ПЭ & 6,5 & 8,5 & 2,0 & 30,8 & & ПК & 7,5 & 7,7 & 0,2 & 2,7 \\
\hline & ПММА & 6,4 & 8,3 & 1,9 & 29,7 & \multirow{3}{*}{45} & ПЭ & 9,7 & 10,0 & 0,3 & 3,0 \\
\hline & ПП & 4,4 & 4,8 & 0,4 & 9,1 & & ПММА & 4,2 & 4,4 & 0,2 & 4,8 \\
\hline & ПК & 9,0 & 8,6 & $-0,4$ & $-4,4$ & & ПК & 8,7 & 9,0 & 0,3 & 3,4 \\
\hline \multirow{3}{*}{26} & ПЭ & 8,7 & 9,9 & 1,2 & 13,8 & 50 & ПММА & 5,2 & 5,6 & 0,4 & 7,7 \\
\hline & ПММА & 7,8 & 9,5 & 1,7 & 21,8 & 52,5 & ПММА & 5,9 & 6,3 & 0,4 & 6,8 \\
\hline & ПП & 5,6 & 6,7 & 1,1 & 19,6 & 55 & ПММА & 6,8 & 7,3 & 0,5 & 7,3 \\
\hline 28 & ПП & 7,9 & 9,4 & 1,5 & 19,0 & 57,5 & ПММА & 7,9 & 8,6 & 0,7 & 8,9 \\
\hline
\end{tabular}

Согласно полученным данным, для полимеров, наполненных УНТ, рассматриваемые величины $\delta \omega$ не превышают $8,9 \%$ (композиты на основе ПММА). При этом сравнительно большие значения $\delta \omega$ для данного композита имеют место лишь при относительно высоких величинах их коэффициентов теплопроводности $(\lambda \geq 50 \mathrm{BT} /$
$(\mathrm{M} \cdot \mathrm{K}))$. Для композитов же на основе ПК и ПЭ максимальные значения $\delta \omega$ заметно ниже и равны соответственно 5,9\% и 4,9\%.

Таким образом, как следует из результатов выполненных исследований, в целом ряде ситуаций при определении массовой доли наполнителей полимерных композитов с заданными 
теплопроводящими свойствами необходимо принимать во внимание зависимость от температуры их коэффициентов теплопроводности. Это особенно важно для всех полимеров, кроме ПК, наполненных микрочастицами алюминия, а также для ПММА, наполненного УНТ.

Остановимся вкратце на обсуждении вопроса, касающегося механизмов теплопроводности в рассматриваемых композитах на примере полимеров, наполненных УНТ.

Применение УНТ в качестве наполнителей при разработке высокотеплопроводных полимерных нанокомпозитов связано, как известно, с аномально высокими теплопроводящими свойствами нанотрубок. Так, согласно данным [10] при комнатной температуре значения коэффициента теплопроводности для одиночных однослойных УНТ находятся в пределах 2800... ...6000 Вт/(м·К). Однако коэффициенты теплопроводности полимеров, наполненных УНТ, оказываются относительно низкими в сравнении с их значениями для собственно УНТ. В случае рассматриваемых композитов значения $\lambda$ не превышают $60 \mathrm{Bт} /(\mathrm{M} \cdot \mathrm{K})$ при массовой доле нанотрубок, равной $10 \%$.

В полимерных нанокомпозитах доминирующим, как известно, является фононный механизм теплопереноса, связанный с тепловыми колебаниями решетки. Согласно современным представлениям серьезными ограничениями для эффективного фононного переноса в полимерах, наполненных УНТ, являются следующие [11]. Во-первых, неравномерность распределения УНТ в полимерной матрице, что не позволяет образовывать достаточное количество прямых контактов между нанотрубками, которые в большой мере ответственны за транспорт фононов. Во-вторых, значительные термические сопротивления в месте контакта нанотрубок также препятствуют переносу фононов (рис. 4). И, наконец, эффективный транспорт фононов затруднен ввиду значительных межфазных термических сопротивлений между УНТ и окружающей полимерной матрицей. Таким образом, решающее значение в получении высокотеплопроводящих нанокомпозитов имеет качество и количество УНТ взаимосвязей, что обеспечивается, прежде всего, за счет относительно большого содержания УНТ и высокого уровня равномерности их распределения в полимере. Выполненные исследования подтверждают данные положения (см., например, [8, 12]).

Следует также отметить, что улучшению качества взаимосвязей УНТ в нанокомопозитах может служить также множество других факторов, роль которых в повышении теплопроводящих свойств полимеров, наполненных УНТ, подлежит дальнейшим исследованиям.

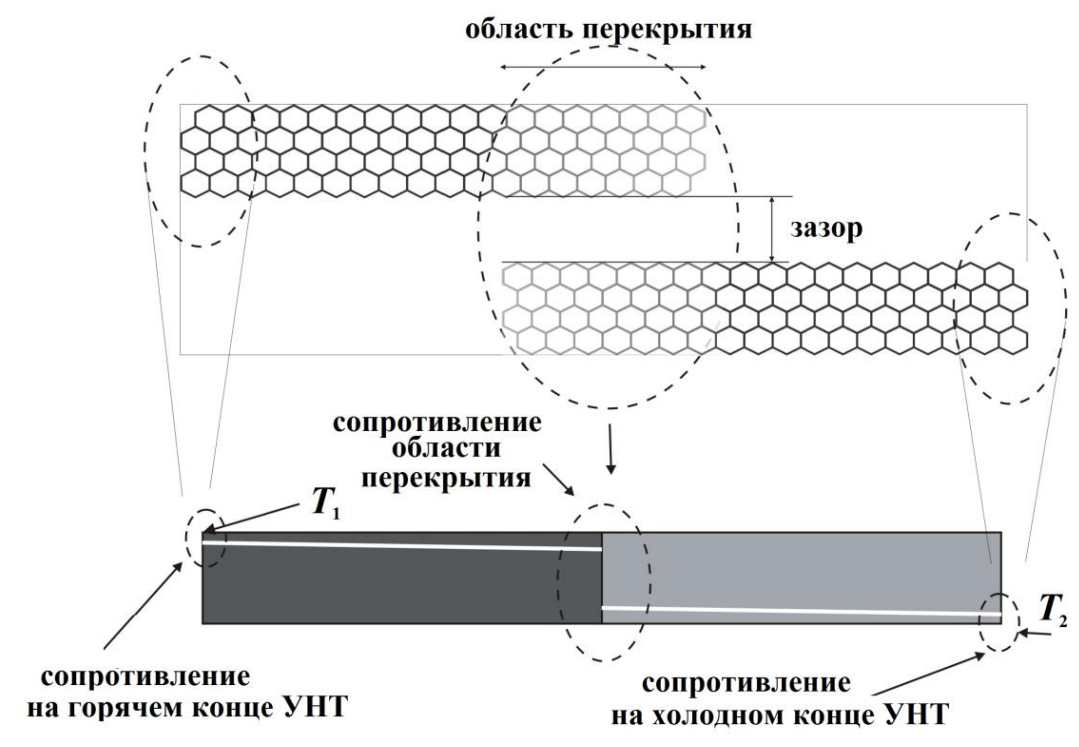

Рис. 4. Схема контакта прилежащих друг к другу углеродных нанотрубок. 


\section{Выводы}

1. Выполнены экспериментальные исследования по определению температурных зависимостей коэффициентов теплопроводности композитов на основе полиэтилена, полиметилметакрылата, полипропилена и поликарбоната при использовании в качестве наполнителей микрочастиц алюминия или УНТ. Показано, что в рассматриваемых температурных интервалах, отвечающих диапазонам рабочих температур этих композитов, величины изменения значений коэффициентов теплопроводности композитов зависят от типа полимерной матрицы и наполнителя, а также от массовой доли последнего. В частности, установлено, что величины указанного изменения являются более существенными при наполнении полимеров микрочастицами алюминия и достигают примерно $10 \%$.

2. Для полимерных микро- и нанокомпозитов с заданными теплопроводящими свойствами проведен сравнительный анализ данных об их составах, полученных с учетом и без учета температурных зависимостей коэффициентов теплопроводности. Установлено, что традиционный подход к определению таких составов по значениям коэффициентов теплопроводности композитов при температуре окружающей среды может приводить к недопустимым погрешностям. В случае использования в качестве наполнителя микрочастиц алюминия его массовые доли, найденные по значениям $\lambda$ при температуре окружающей среды, могут быть заниженными более, чем на $30 \%$ в сравнении с уточненными величинами этих долей, при определении которых принимались во внимание температурные зависимости коэффициентов теплопроводности композитов.

3. Рассмотрены вопросы, касающиеся механизмов теплопроводности в полимерах, наполненных УНТ. Отмечается, что к основным факторам, препятствующим эффективному транспорту фононов, относятся: неравномерность распределения УНТ в полимере, а также наличие значительных термических сопротивлений как в месте контакта нанотрубок, так и между трубками и полимерной матрицей.

\section{ЛИТЕРАТУРА}

1. Yimin $Y$. The effect of interfacial state on the thermal conductivity of functionalized $\mathrm{Al}_{2} \mathrm{O}_{3}$ filled glass fibers reinforced polymer composites / Y. Yimin, Z. Xiaoliang, G. Kun, S. Rong, X. Jian-bin // Composites: Part A. - 2015. - Vol. 69. - P. 49-55.

2. Davide P. Graphene-based coatings on polymer films for gas barrier applications / P. Davide, Y.X. Zhen, Ch. Meganne, L. Simone, M. Matteo, M. Vittorio, D. Ferruccio, P. Vincenzo // Carbon. - 2015. - P. 1-35.

3. Ramanathan T. Functionalized graphene sheets for polymer nanocomposites / T. Ramanathan, A.A. Abdala, S. Stankovich, D.A. Dikin, M. HerreraAlonso, R.D. Piner, D.H. Adamson, H.C. Schniepp, X. Chen, R.S. Ruoff, S.T. Nguyen, I.A. Aksay, R.K. Prud'homme, L.C. Brinson // Nature. - 2008. Vol.3. - P. 327-331.

4. Chao $W$. Highly Conductive Nanocomposites with Three- Dimensional, Compactly Interconnected Graphene Networks via a Self-Assembly Process / Wu Chao, Huang Xingyi, Wang Genlin, L. Libing, C. Gan, Li Guangyv, J. Pingkai // Advanced functional material. - 2012. - P. $1-8$.

5. Kim G. High thermal conductivity in amorphous polymer blends by engineered interchain interactions / Gun-Ho Kim, Dongwook L, Apoorv Shanker, Lei Shao, Min Sang Kwon, David Gidley, Jinsang Kim, Kevin P. P. // Nature Materials. - 2014. - P. 1-6.

6. Rafael A. Functionalization of Natural Graphite for Use as Reinforcement in Polymer Nanocomposites / Rafael Araujo, Maria F. V. Marques, Renato Jonas, Iryna Grafova, Andriy Grafov // Journal of Nanoscience and Nanotechnology. 2015. - Vol. 15. - P. 6176-6182.

7. Carlos V. O. Reinforcement and toughening mechanisms in polymer nanocomposites Carbon nanotubes and aluminum oxide / Carlos V. Opelt, Daniela Becker, Carlos M. Lepienski, Luiz A.F. Coelho // Composites: Part B. - 2015. - Vol. 75. - P. 119-126.

8. Долинский A.A. Влияние методов получения полимерных микро- и нанокомпозитов на их теплофизические свойства / Долинский А.А., Фиалко Н.М., Динжос Р.В., Навродская Р.А. // Промышленная теплотехника. - 2015. - №4. C. 5-13. 
9. Giovanni A. L. A Steady-State Apparatus to Measure the Thermal Conductivity of Solids / A. L.Giovanni // Int. J Thermophys. - 2008. - 29. P.664-677.

10. Lindsay $L$. Lattice thermal conductivity of single-walled carbon nanotubes: beyond the relaxation time approximation and phononphonon scattering selection rules / L. Lindsay, D.A. Broido, N. Mingo // Phys. Rev. B. - 2009. Vol. 80. - P. 125407/1-125407/7.

\section{TEMPERATURE DEPENDENCE OF THE CONDUCTIVE COEFFICIENTS OF POLYMER MICRO- AND NANOCOMPOSITES FOR HEAT-EXCHANGE APPARATUS}

\section{Dolinsky A.A. ${ }^{1}$, Fialko N.M. ${ }^{1}$, Dinzhos R.V. ${ }^{2}$, Navrodskaya R.A. ${ }^{1}$}

${ }^{1}$ Institute of Engineering Thermophysics, National Academy of Sciences of Ukraine, 2a, Zhel-yabova str., Kyiv, 03680, Ukraine

${ }^{2}$ Nikolaev National University. named after V.A. Sukhomlinskiy, 24, Nikolska str., Mykolaev, 540030, Ukraine

The results of experimental investigations of the temperature dependence of the heat-conductivity coefficients of polymer micro- and nanocomposites for the manufacture of heat exchange equipment are given. The respective dependences for composites based on polyethylene, polymethylmethacrylate, polypropylene and polycarbonate, filled with carbon nanotubes and alumina microparticles are considered. The data relating to the account of this dependence in determining the composition of composites with desired heat-conducting properties are presented. The analysis of the possibilities of determining such compositions using minimum
11. Han Z., Thermal conductivity of carbon nanotubes and their polymer nanocomposites: a review / Z. Han, A. Fina // Prog. Polym. Sci. - 2011. - Vol. 36. - P. 914-944.

12.Долинский А.А. Теплофизические свойства низкотеплопроводных полимерных нанокомпозитов для элементов энергетического оборудования / А.А. Долинский, Н.М. Фиалко, Р.В. Динжос, Р.А. Навродская // Промышленная теплотехника. - 2015. - № 6. - С. 5-16.

and maximum temperature of the test temperature ranges is performed. The features of the heat transfer mechanisms in polymers filled with carbon nanotubes are discussed.

Reference 12, figures 4, tables 2 .

1. Yimin $Y$. The effect of interfacial state on the thermal conductivity of functionalized $\mathrm{Al}_{2} \mathrm{O}_{3}$ filled glass fibers reinforced polymer composites $/$ Yimin Y., Xiaoliang Z., Kun G., Rong S., Jian-bin X. Composites: Part A. - 2015. - Vol. 69. - P. 49-55.

2. Davide P. Graphene-based coatings on polymer films for gas barrier applications / Davide P., Zhen Y.X., Meganne Ch., Simone L., Matteo M., Vittorio M., Ferruccio D., Vincenzo P. - Carbon 2015. - P. 1-35.

3. Ramanathan T. Functionalized graphene sheets for polymer nanocomposites / T. Ramanathan, A.A. Abdala, S. Stankovich, D.A. Dikin, M. HerreraAlonso, R.D. Piner, D.H. Adamson, H.C. Schniepp, X. Chen, R.S. Ruoff, S.T. Nguyen, I.A. Aksay, R.K. Prud'homme, L.C. Brinson - Nature. - 2008. - Vol.3. - C.327-331.

4. Chao $W$. Highly Conductive Nanocomposites with Three- Dimensional, Compactly Interconnected Graphene Networks via a Self-Assembly Process / Chao Wu, Xingyi Huang, Genlin Wang, Libing L., Gan C., Guangyv Li, Pingkai J. - Advanced 
functional material. - 2012. - P. $1-8$.

5. Kim G. High thermal conductivity in amorphous polymer blends by engineered interchain interactions / Gun-Ho Kim, Dongwook L, Apoorv Shanker, Lei Shao, Min Sang Kwon, David Gidley, Jinsang Kim, Kevin P. P. - Nature Materials. - 2014. - P. $1-6$.

6. Rafael A. Functionalization of Natural Graphite for Use as Reinforcement in Polymer Nanocomposites / Rafael Araujo, Maria F. V. Marques, Renato Jonas, Iryna Grafova, Andriy Grafov - Journal of Nanoscience and Nanotechnology. - 2015. - Vol. 15. - P. 6176-6182.

7. Carlos V. O. Reinforcement and toughening mechanisms in polymer nanocomposites Carbon nanotubes and aluminum oxide / Carlos V. Opelt, Daniela Becker, Carlos M. Lepienski, Luiz A.F. Coelho-Composites Part B. - 2015. - Vol. 75. - P. 119-126.

8. Dolinsky A.A. Influence of preparation methods of polymer micro- and nanocomposites on their thermophysical properties / A.A. Dolinskiy,
N.M. Fialko, R.V. Dinzhos, R.A. Navrodskaya // Promyshlennaja teplotechnica. - 2015. - №4. - P. 5-13.

9. Giovanni A. L. A Steady-State Apparatus to Measure the Thermal Conductivity of Solids. // Int J Thermophys 2008; 29:664-677.

10. Lindsay L. Lattice thermal conductivity of single-walled carbon nanotubes: beyond the relaxation time approximation and phonon-phonon scattering selection rules // Phys. Rev. B. - 2009. Vol. 80. - P. 125407/1-125407/7.

11.Han Z. Thermal conductivity of carbon nanotubes and their polymer nanocomposites: a review// Prog. Polym. Sci. - 2011. - Vol. 36. - P. 914-944.

12.Dolinsky A.A. Thermophysical properties low thermal conductivity of polymer nanocomposites for the elements of power equipment / A.A. Dolinskiy, N.M. Fialko, R.V. Dinzhos, R.A. Navrodskaya // Promyshlennaja teplotechnica.2015. - № 6. - P. 5-16.

Получено 04.01.2016 Received 04.01.2016 